Bundesgesundheitsbl 2012 $55: 1-2$

DOI 10.1007/s00103-011-1398-7

Online publiziert: 24. Dezember 2011

(c) Springer-Verlag 2011

I. Pigeot · R. Foraita

Bremer Institut für Präventionsforschung und Sozialmedizin, Bremen

\title{
Faul macht krank?
}

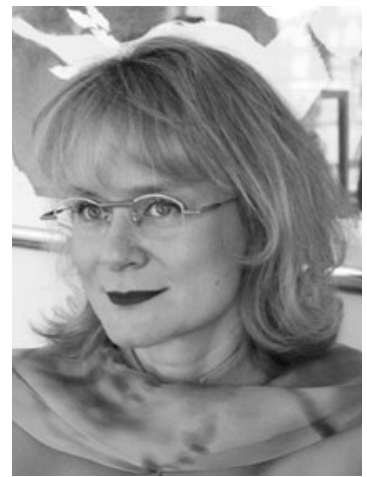

gungsapparat: „Sport beugt Gelenkerkrankungen und Rückenschmerzen vor. " Damit sind aber noch längst nicht alle positiven Auswirkungen von Bewegung auf die Gesundheit abgehandelt, zeigen sich doch auch positive Effekte von ausreichender körperlicher Aktivität auf die Entstehung und den Verlauf von Krebserkrankungen und auf allgemeine Alterungsprozesse. Betrachtet man darüber hinaus noch den $\mathrm{Zu}$ sammenhang zwischen körperlicher Aktivität und Übergewicht beziehungsweise Adipositas, so sind die positiven gesundheitlichen Auswirkungen ausreichender Bewegung gar nicht hoch genug einzuschätzen. Schließlich hängen an der Fettleibigkeit dermaßen viele Komorbiditäten wie Diabetes, Gelenkprobleme, Herz-Kreislauf-Erkrankungen, um nur einige zu nennen, dass alleine eine Reduktion des Übergewichts durch mehr Bewegung zu einer deutlichen Steigerung der Gesundheit jedes Einzelnen, aber auch im Sinne von Public Health führen würde.

Im Zusammenhang mit der Diskussion um die positiven Auswirkungen von ausreichender Bewegung auf die Gesundheit wird immer stärker darauf hingewiesen, dass es sich dabei nicht um aufwendige sportliche Aktivitäten handeln muss, sondern dass sie auch einfach in den Alltag eingebunden werden kann, indem man zum Bei-

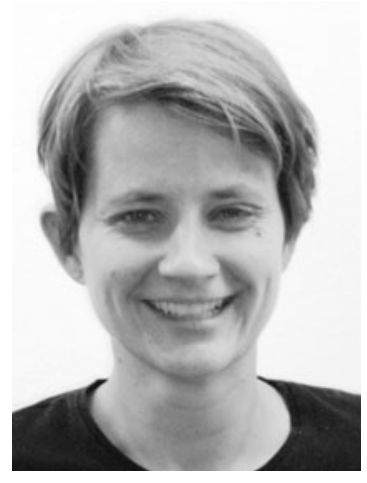

spiel Einkäufe mit dem Rad oder zu Fuß erledigt oder den Aufzug bzw. die Rolltreppe zugunsten der Treppe verschmäht.

Das vorliegende Heft des Bundesgesundheitsblattes widmet sich mehreren Fragen, die sich um diesen Themenkomplex ansiedeln. So wird die wissenschaftliche Evidenz für die Wirkung von körperlicher Aktivität auf verschiedene Erkrankungen diskutiert, aber auch die methodische Grundlage zur Messung von körperlicher Aktivität herausgearbeitet. Die korrekte Messung körperlicher Aktivität ist unter anderem wichtig für die Herleitung evidenzbasierter Empfehlungen für ein gesundheitsförderndes Maß an körperlicher Bewegung. Sie spielt aber auch eine Rolle in der Beurteilung der Effektivität von Interventionsmaßnahmen zur Steigerung der körperlichen Aktivität, die ebenfalls Thema verschiedener Artikel in diesem Heft sind. Darüber hinaus werden Fakten zum Bewegungsverhalten der deutschen Bevölkerung speziell in Bezug zu gängigen Leitlinien beziehungsweise Empfehlungen zur körperlichen Aktivität reflektiert.

Im Einzelnen werden die oben genannten Bereiche wie folgt durch die Beiträge in diesem Heft abgedeckt:

In dem ersten Artikel von Ulrich et al. geht es darum, am Beispiel von Krebserkrankungen die physiologischen und mo- 
lekularen Wirkmechanismen von körperlicher Aktivität auf deren Entstehung und Verlauf aufzuzeigen, wodurch die Kausalität dieses Zusammenhangs untermauert wird. Auch wenn nicht in jedem Einzelfall in diesem Heft die genauen Wirkmechanismen dargestellt sind, so wird dennoch in den weiteren Beiträgen zu den verschiedenen Krankheiten die jeweilige Evidenz herausgearbeitet. So diskutieren Steindorf et al. in ihrem Artikel, dass eine erhöhte körperliche Aktivität das Risiko für Kolonkrebs reduziert, ein solcher Effekt für Endometrium- und postmenopausalen Brustkrebs wahrscheinlich ist und für prämenopausalen Brustkrebs, Prostata-, Lungen- und Pankreaskrebs vermutlich vorliegt. Den Stand der Forschung zum Zusammenhang von körperlicher Inaktivität und dem Auftreten kardiovaskulärer Erkrankungen fassen Hansel et al. in ihrem Beitrag zusammen. Auf Basis epidemiologischer Studien geben Winkler et al. eine entsprechende Beurteilung der Assoziation zwischen körperlicher Aktivität und Übergewicht bzw. Adipositas, dabei widmen sie sich insbesondere dem Problem der Erfassung der körperlichen Aktivität, die gerade in epidemiologischen Studien eine Herausforderung darstellt. Des Weiteren geben Herrmann et al. einen ausführlichen Überblick zur Evidenz des Zusammenhangs von körperlicher Aktivität und Knochengesundheit bereits im Kindes- und Jugendalter, für das sich ein erhöhter Knochenzuwachs bei entsprechender körperlicher Aktivität nachweisen lässt. Der schon in der Überschrift angesprochene Effekt von körperlicher Aktivität auf die psychische Gesundheit wird in dem Beitrag von Schulz et al. wieder aufgegriffen und im Detail behandelt. Schließlich geben Abu-Omar und Rütten einen Einblick in die Thematik aus der Public-Health-Perspektive. Sie diskutieren Empfehlungen für ein adäquates Maß an körperlicher Aktivität und stellen erste Prävalenzen dar. $\mathrm{Zu}$ dem beschreiben sie verschiedene Typen von Interventionsmaßnahmen speziell in diesem Bereich.

Der letztgenannte Aspekt, insbesondere mit Blick auf primärpräventive Maßnahmen, ist Gegenstand des Beitrags von Jordan et al. In diesem werden unter anderem konkrete Beispiele für Präventionsprogramme gegeben, und es wird eine Einschätzung der aktuellen Präventionsland- schaft zur Förderung körperlicher Aktivität getroffen. Ein spezielles Programm, das sich in diesen Kontext einbetten lässt, dient der sportmedizinischen Bewegungsberatung bei Arbeitslosen und wird von Gabrys et al. vorgestellt.

Der Beitrag von Rosenbaum widmet sich der bereits oben angesprochenen wichtigen Thematik der "richtigen“ quantitativen Erfassung körperlicher Aktivität. Verschiedene Messmethoden und ihre möglichen Einsatzgebiete werden vorgestellt und bewertet; dabei zeigt sich, dass die Messung körperlicher Aktivität kein leichtes Unterfangen ist. Allerdings erweist sich körperliche Fitness als eine besser zu messende Grö$\beta e$, wie Brandes in seinem Beitrag zeigt. Er reflektiert zudem kritisch den bedeutsamen Zusammenhang zwischen körperlicher Aktivität und körperlicher Fitness sowie deren Effekt auf die Gesundheit. In der Studie „Gesundheit in Deutschland aktuell 2009 " wurde die körperliche Aktivität über einen Fragebogen bei den Teilnehmern erfasst. Auf Basis dieser Daten geben Lampert et al. eine ausführliche Darstellung der Verbreitung von körperlicher und sportlicher Aktivität in Deutschland unter verschiedenen Gesichtspunkten, zum Beispiel unter Berücksichtigung zeitlicher Trends. Basierend auf dem Kinder- und Jugendgesundheitssurvey, geben Krug et al. eine Einschätzung zum Zusammenhang zwischen körperlicher Aktivität und dem allgemeinen Gesundheitszustand bei Kindern und Jugendlichen.

In der Hoffnung, dass Ihnen die Beiträge in Ihrer ärztlichen Beratungstätigkeit nützlich sind beziehungsweise Ihnen direkt Lust auf Bewegung vermittelt haben, verbleiben wir

Ihre

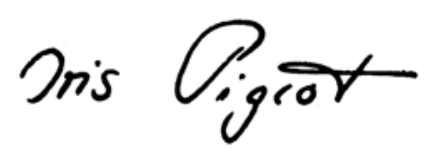

Iris Pigeot

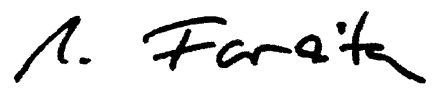

Ronja Foraita

\section{Korrespondenzadressen}

Prof. Dr. I. Pigeot

Bremer Institut für Präventionsforschung und Sozialmedizin

Achterstraße 30, 28359 Bremen

pigeot@bips.uni-bremen.de

\section{Dr. R. Foraita}

Bremer Institut für Präventionsforschung und Sozialmedizin

Achterstraße 30, 28359 Bremen

foraita@bips.uni-bremen.de 